\title{
Crecimiento del gato doméstico mediante un modelo logístico
}

\author{
Domestic cat growth through a logistic model \\ Pere Miquel Parés-Casanova ${ }^{1,3}$, Arcesio Salamanca-Carreño ${ }^{2}$, René Alejandro \\ Crosby-Granados ${ }^{2}$, Karen Viviana Santos-Diaz ${ }^{2}$
}

\section{Resumen}

El objetivo del estudio fue describir y analizar el patrón de crecimiento del gato doméstico. Se trabajó con 68 gatos (37 machos y 31 hembras, con rango de edades de 1.484 y 1.5-84 meses, respectivamente), de composición racial indefinida, del área urbana del municipio de Arauca, Colombia. Se registró la edad, longitud corporal, circunferencia pelviana, circunferencia del cráneo, longitud del miembro posterior y longitud del miembro anterior derecho. Se determinó el crecimiento para cada sexo con la expresión logística $y=a /(1+b e-c x)$. Los resultados mostraron un patrón de crecimiento similar en ambos sexos, excepto la del miembro posterior $(\mathrm{p}=0.033)$, que presentó un crecimiento acelerado inicial y un posterior crecimiento asintótico en las hembras, pero con poco cambio etario en los machos. La talla final fue similar para ambos sexos. Este trabajo proporciona una primera aproximación sobre los patrones de crecimiento del gato doméstico.

Palabras clave: Arauca; modelo de crecimiento; peso corporal; tamaño corporal; tasa de crecimiento

\section{AbSTRaCT}

The objective of the study was to describe and analyze the growth pattern of the domestic cat. A total of 68 cats (37 males and 31 females, with an age range of 1.4-84 and 1.5-84 months, respectively), of undefined racial composition, from the urban area of the municipality of Arauca, Colombia were studied. Age, body length, pelvic girth, skull perimeter, and fore and hind right limb lengths were recorded. Growth was determined for

${ }^{1}$ Departament de Ciència Animal, Universitat de Lleida, Cataluña, España

${ }^{2}$ Facultad de Medicina Veterinaria y Zootecnia, Universidad Cooperativa de Colombia, Arauca, Colombia

${ }^{3}$ E-mail: peremiquelp@ca.udl.cat

Recibido: 20 de marzo de 2018

Aceptado para publicación: 31 de agosto de 2018 
each sex with the logistic equation $y=a /(1+b e-c x)$. Results showed a similar growth pattern between sexes, except for hind limb length $(\mathrm{p}=0.033)$, which presented an initial accelerated growth with an ulterior asymptotic growth in females, but there were few changes in males. Final size was similar for both sexes. This study constitutes a first contribution to growth models in domestic cats.

Key words: Arauca; growth model; body weight; body size; growth rate

\section{INTRODUCCIÓN}

El gato doméstico (Felis catus, Linnaeus 1758) es el mamífero más especializado y evolucionado de todos los carnívoros, y se cree que se originó a partir del gato silvestre (Felis silvestris subspp) (Lipinski et al., 2008; Sañudo, 2009). Los cruces continuos y la creación de nuevas razas han contribuido a cambios en sus características generales (Lipinski et al., 2008; Sañudo, 2009).

Para analizar las diferencias entre especies y dentro de una especie es fundamental la descripción anatómica y morfológica. La morfometría en los gatos domésticos es un tema del cual existen escasos estudios, razón por la que no encuentran medidas estándares para estos animales (Sotillo y Serrano, 1985).

Cuando se consideran las diferentes partes o tejidos de un organismo, no todas crecen con la misma intensidad y ritmo, lo que origina un desarrollo diferencial. Por lo tanto, otro concepto íntimamente ligado al de desarrollo es el de desarrollo relativo o alométrico. El principio de la alometría es que los cambios morfogenéticos que se producen en un animal en desarrollo tienen lugar, principalmente, por desarrollo relativo, o sea, por el desarrollo que se produce en las distintas partes del organismo animal como un todo (Gould, 1966; Voje et al., 2014; Ávila, 2017).

El uso de las funciones matemáticas para describir el crecimiento animal permite- identificar «puntos estratégicos», así como describir el desarrollo corporal como una función de la edad del animal (France et al., 1996). El contar con un modelo adecuado para la curva de crecimiento de los animales domésticos es el primer paso en la predicción de los requerimientos nutricionales para las diferentes razas (France et al., 1996; Domínguez-Viveros et al., 2013; Marinho et al., 2017). Varios modelos matemáticos no lineales son usados para describir el crecimiento en los animales. La idea subyacente es que la tasa de cambio está relacionada con el tamaño. La relación cuadrática resultante implica que al inicio de su vida, cuando tienen tamaños muy pequeños, los individuos crecen moderadamente rápido; conforme crecen, sus tasas de crecimiento se aceleran hasta alcanzar una máxima velocidad a tallas relativamente intermedias; $y$ después de este máximo, la velocidad de crecimiento disminuye de manera no lineal conforme aumenta la talla y se aproxima a una asíntota (Paine $e t$ al., 2012). La función logística permite apreciar mucho mejor lo que ocurre durante el crecimiento, ya que describe de manera más precisa que otras, como la exponencial, lo que realmente ocurre con los seres vivos (Motulsky y Christopoulos, 2003; Paine et al., 2012).

El objetivo del estudio fue describir y analizar el patrón de crecimiento del gato doméstico, específicamente en identificar el patrón de crecimiento y las posibles diferencias entre machos y hembras en el crecimiento corporal. 
Cuadro 1. Expresiones $y=a /\left(1+b^{-c x}\right)$ por el sexo de los gatos y para cada una de las variables analizadas (37 machos de 1.4-84 meses, 31 hembras de 1.5-84 meses)

\begin{tabular}{lllc}
\hline Variable & Machos & Hembras & $P^{1}$ \\
\hline Peso & $5.00 /\left(1+0.86^{-0.01 x}\right)$ & $4.00 /\left(1+1.03^{-0.04 x}\right)$ & 0.573 \\
Longitud corporal & $60.06 /\left(1+0.58^{-0.005 x}\right)$ & $53.05 /\left(1+0.93^{-0.02 x}\right)$ & 0.101 \\
Circunferencia pelviana & $54.05 /\left(1+0.86^{-0.001 x}\right)$ & $52.05 /\left(1+0.70^{0.002 x}\right)$ & 0.324 \\
Circunferencia del cráneo & $50.05 /\left(1+0.83^{-0.005 x}\right)$ & $38.04 /\left(1+0.71^{-0.02 x}\right)$ & 0.125 \\
Longitud miembro anterior & $28.03 /\left(1+0.54^{-0.03 x}\right)$ & $30.03 /\left(1+0.40^{-0.005 x}\right)$ & 0.705 \\
Longitud miembro posterior & $42.04 /\left(1+0.72^{-0.005 x}\right)$ & $30.03 /\left(1+0.70^{-0.04 x}\right)$ & 0.033 \\
\hline
\end{tabular}

El valor $p$ corresponde al ANCOVA. Diferencia significativa entre sexos para la longitud del miembro posterior

Medidas corporales en $\mathrm{cm}$, excepto para el peso, expresado en $\mathrm{kg}$

\section{Materiales y Métodos}

Los datos fueron recolectados en el área urbana del municipio de Arauca, Colombia. Se estudiaron 68 animales, con rango de edad para los machos $(n=37)$ de $1.4-84$ meses y para las hembras $(n=31)$ de $1.5-84$ meses. Los animales eran de composición racial indefinida, procedentes de hogares particulares, donde se les tiene como mascotas o animales de compañía y para el control biológico de roedores y otras especies de animales.

De cada animal se obtuvieron los siguientes datos: edad (declarada por los propietarios), longitud corporal (medida desde la articulación sacro-coccígea a la nariz), circunferencia pelviana (anterior a las alas del íleon), circunferencia del cráneo, longitud del miembro posterior derecho (de la cabeza del fémur a maléolo lateral de la tibia) y longitud del miembro anterior derecho (de la articulación escápulo-humeral a la articulación radio carpo cubital). Las medidas se obtuvieron con cinta métrica inextensible, según procedimiento de López (López, 2008), quienes presentan una descripción detallada de los puntos de medida. El peso fue obtenido con báscula digital con capacidad para $5 \mathrm{~kg}$. La manipulación de los animales fue minimizada e incruenta. En ningún caso se recurrió a contenciones agresivas o químicas.

En el análisis estadístico se utilizó el test $D$ de Kolmogorov-Smirnov para evaluar la igualdad en la distribución de los sexos por edad. Se calcularon para cada una de las variables las expresiones $y=a /\left(1+b^{-c x}\right)$. Para comparar entre sexos se recurrió a un análisis de covarianza (ANCOVA). Todos los análisis estadísticos fueron realizados mediante la aplicación estadística PAST v. $2.17 \mathrm{c}$ (Hammer et al., 2001), a un valor p de 5\%.

\section{Resultados}

La distribución etaria para ambos sexos no fue significativamente diferente $(D=0.238$, $\mathrm{p}=0.253)$. En el Cuadro 1 aparecen las expresiones $y=a /\left(1+b^{-c x}\right)$ por sexo y para cada una de las variables analizadas, junto con los resultados del ANCOVA. En la Figura 1 aparecen los modelos logísticos para la longitud del miembro posterior, según sexo, donde se reflejan diferencias significativas en la longitud del miembro posterior $(\mathrm{p}<0.05)$. 

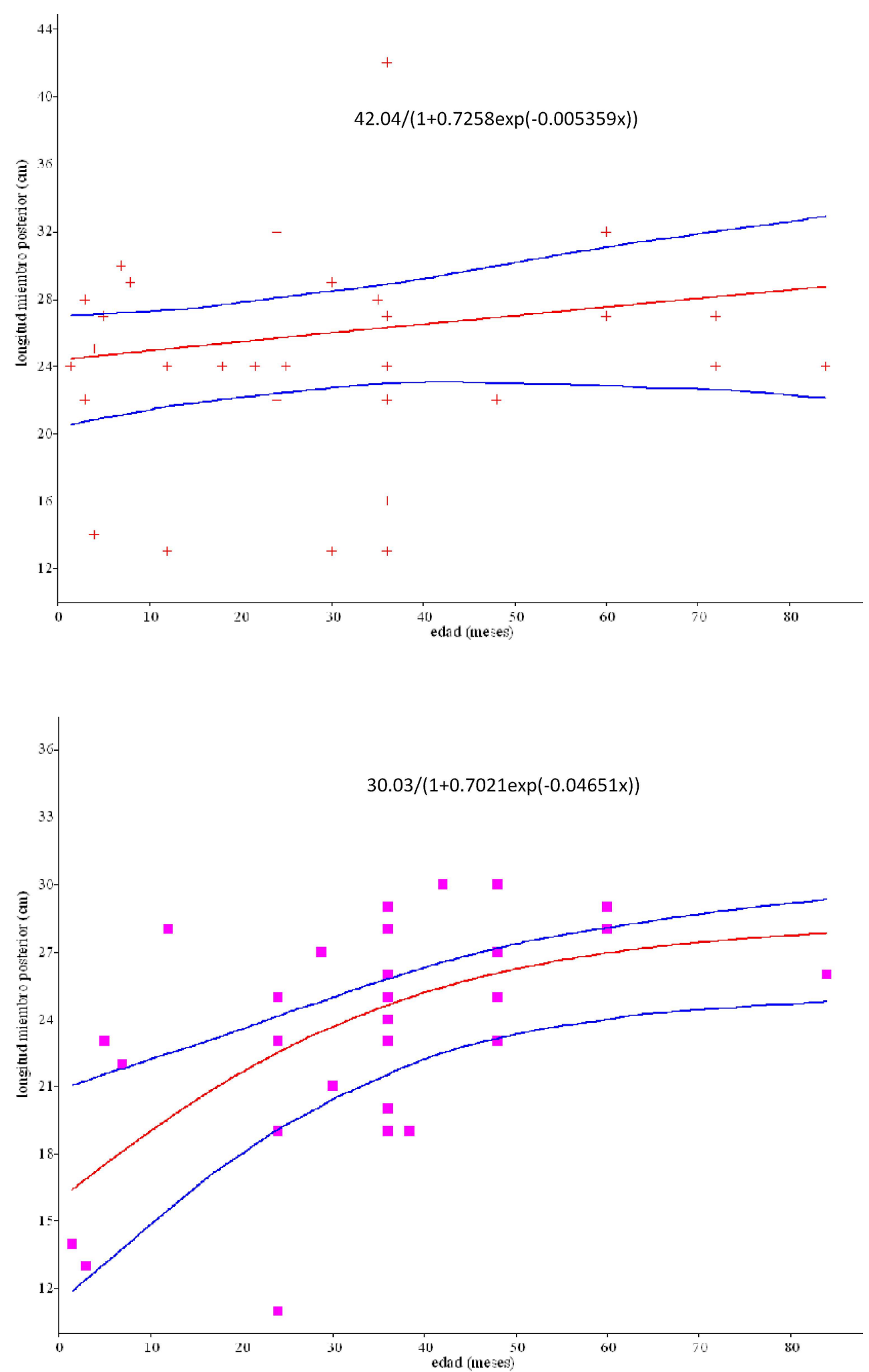

Figura 1. Regresión por el modelo logístico de la longitud del miembro posterior para machos ( $\mathrm{n}=37$, cruces) y hembras ( $\mathrm{n}=31$, cuadrados rellenos). Las líneas azules corresponden al intervalo de confianza del $95 \%$ 


\section{Discusión}

La modificación de las proporciones de las diversas regiones morfológicas, órganos y sistemas, hasta que se accede al estado adulto o estable, es el resultado del crecimiento desigual o relativo de las diferentes partes del organismo, donde los modelos más idóneos para su cuantificación son los no lineales.

En el presente trabajo se pretende describir las características ontogénicas del peso de determinados elementos óseos del gato Araucano, a partir del 1.5 meses de edad y hasta bien superada la plena madurez. En la expresión gráfica de la fórmula logística puede detectarse un crecimiento inicial relativamente muy rápido, que se expresa cuantitativamente como proporcional al tamaño del organismo. Esta forma de crecimiento es frecuentemente llamada 'crecimiento exponencial'. En una segunda etapa, el crecimiento es relativamente menos rápido, ya que mucha de la energía del organismo es dedicada a mantener su actual tamaño, y durante esta etapa el crecimiento del organismo puede ser aproximado por la relación lineal. El crecimiento disminuye por un balance entre la energía del organismo y los requerimientos de conservación. El tamaño del organismo en esta tercera etapa sería asintótico, tendiendo al límite superior sobre el tamaño. Así, el escalamiento ontogénico en las extremidades posteriores presenta una diferenciación sexual, en el que comparativamente en las hembras crece más rápido que en los machos; es decir, las hembras tienden a ser más «paticortas por detrás» que los machos a una edad primeriza.

No se encontraron diferencias significativas en el resto de las variables estudiadas. Siendo la parte asintótica similar para ambos sexos para todas las variables, se deduce igualmente que en la madurez, tanto machos como hembras tendrán peso, perímetros y longitudes similares, y por ende un dimorfismo sexual nulo para estas variables.
La tasa de crecimiento puede ser contemplada desde dos perspectivas que no son mutuamente excluyentes. Por un lado, como una combinación entre la disponibilidad de recursos, el ambiente térmico, las restricciones biofísicas sobre el tiempo disponible de forrajeo y la capacidad para procesar alimento. Por otro lado, el componente genético, que puede determinar en gran medida la trayectoria del crecimiento y su relación con las demás características de historia de vida (Zamora-Abrego et al., 2012; DomínguezViveros et al., 2013).

Se sugiere que las diferencias de crecimiento entre sexos para la longitud del miembro posterior deben ser reflejo de adaptaciones locales a condiciones ambientales específicas o a un particular estilo de vida, más que un proceso fisiológico propio de la especie. Los huesos cumplen simultáneamente diversas funciones, estando los requerimientos estructurales sujetos al comportamiento y locomoción (Doube et al., 2009). El grado de dimorfismo sexual depende de muchos factores, pero uno de los principales se relaciona a la malnutrición proteico-calórica, que actúa reduciendo las diferencias en tamaño entre los sexos (Frynta et al., 2012). En la especie humana, en caso de estrés nutricional, los hombres son más susceptibles a este estrés nutricional, por lo que reducen su tamaño, mientras que las mujeres son más estables (Pettenati et al., 2002). En el ovino, al ser el macho más inmaduro al momento de nacer, puede ser afectado por una deficiencia nutricional; en cambio la hembra resulta más resistente a penurias alimenticias (Sotillo y Serrano, 1985; Sañudo, 2009; Pineda et al., 2011). Entonces, en condiciones nutricionales subóptimas, como sería el caso del gato Araucano, se expresarían esas diferencias en el desarrollo a favor de la hembra.

Este trabajo proporciona una primera aproximación sobre los patrones de crecimiento del gato doméstico. Los resultados aquí obtenidos muestran un patrón de crecimiento similar en ambos sexos, excepto la 
del miembro posterior, que presenta un crecimiento acelerado inicial y un posterior crecimiento asintótico, en las hembras, y con poco cambio en los machos, aunque la talla final acaba siendo similar para ambos. No obstante, este patrón diferencial no es probablemente propio de la especie.

\section{Conclusiones}

Se presenta un escalamiento ontogénico en las extremidades posteriores del gato, creciendo más rápido en las hembras que en los machos; pero en la madurez, tanto machos como hembras, acaban presentando un peso corporal y medidas de miembros y cabeza similares, y por ende, un dimorfismo sexual nulo.

\section{Agradecimiento}

Los autores agradecen todos los propietarios de animales que permitieron la medición, no siempre fácil.

\section{Literatura Citada}

1. Avila D. 2017. Variaciones alométricas durante el crecimiento en siete especies de garzas (Aves: Ardeidae). Revi Biol Trop 65: 1347-1357.

2. Domínguez-Viveros J, RodríguezAlmeida FA, Núñez-Domínguez $R$, Ramírez-Valverde $R, \quad$ OrtegaGutiérrez JA, Ruiz-Flores A. 2013. Ajuste de modelos no lineales y estimación de parámetros de crecimiento en bovinos tropicarne. Agrociencia 47: 25-34.

3. Doube M, Wiktorowicz-Conroy A, Cristiansen $P$, Hutchinson JR, Shefelbine S. 2009. Three-dimensional geometric analysis of felid limb bone allometry three-dimensional geometric analysis of felid limb bone allometry. Plos One 4: e4742. doi: 10.1371/journal.pone.0004742
4. France J, Dijkstra J, Dhanoa M. 1996. Growth functions and their application in animal science. Annales Zootechnie 45(Suppl. 1): 165-174. doi: 10.1051/animres:19960637

5. Frynta D, Baudyšová J, Hradcová P, Faltusová K, Kratochvíl L. 2012. Allometry of sexual size dimorphism in domestic dog. PLoS One 7(9). doi: 10.1371/journal.pone.0046125

6. Gould SJ. 1966. Allometry and size in ontogeny and phylogeny. Biol Rev Camb Philos Soc 41: 587-638. doi: 10.1111/ j.1469-185X.1966.tb01624.x

7. Hammer Ø, Harper DAT, Ryan PD. 2001. PAST v. $2.17 \mathrm{c}$. Palaeontol Electron 4: 1-229.

8. Lipinski MJ, Froenicke L, Baysac $\mathrm{KC}$, Billings NC, Leutenegger CM, Levy AM, Longeri M, et al. 2008. The ascent of cat breeds: genetic evaluations of breeds and worldwide random bred populations. Genomics 91: 12-21. doi: 10.1016/j.jaad.2014.07.052.A

9. López A. 2008. Determinación de variables morfométricas corporales en 100 felinos en tres clínicas del norte de Bogotá. Tesis de Médico Veterinario. Bogotá, Colombia: Univ. De la Salle. $114 \mathrm{p}$.

10. Marinho KNDS, Freitas AR De Falcão AJDS, Dias FEF. 2013. Nonlinear models for fitting growth curves of Nellore cows reared in the Amazon Biome. R Bras Zootec 42: 645 650. doi : 10.1590/S1516-35982013000900006

11. Motulsky H, Christopoulos A. 2003. Fitting models to biological data using linear and nonlinear regression. A practical guide ro curve fitting. San Diego, CA, USA: GraphPad Software.

12. Paine CET, Marthews TR, Vogt DR, Purves D, Rees M, Hector A, Turnbull LA. 2012. How to fit nonlinear plant growth models and calculate growth rates: an update for ecologists. Methods Ecol Evol 3: 245-256. doi: 10.1111/j.2041210X.2011.00155.X 
13. Pettenati-Soubayroux I, Signoli M, Dutour O. 2002. Sexual dimorphism in teeth: discriminatory effectiveness of permanent lower canine size observed in a XVIIIth century osteological series. Forensic Sci Int 126: 227-232. doi: 10.1016/S0379-0738(02)00080-4

14. Pineda JM, Mujica F, Barra $R$ de la, Blanco JA. 2011. Evaluación zoométrica de la base materna de la raza ovina Chilota comparada con dos razas ovinas predominantes en las regiones de Los Lagos y Los Ríos. Agro Sur 39: 143-156.

15. Sañudo C. 2009. Valoración morfológica de los animales domésticos. Madrid: Ministerio de Medio Ambiente y Medio Rural y Marino. 859 p.
16. Sotillo JL, Serrano V. 1985. Producción animal. I. Etnologia zootecnica. Albacete: Tebar. 402 p.

17. Voje KL, Hansen TF, Egset CK, Bolstad GH, Pélabon C. 2014.

Allometric constraints and the evolution of allometry. Evolution 68: 866-885. doi: 10.1111/evo.12312

18. Zamora-Abrego JG, Zuñiga-Vega JJ, Ortega-León AM. 2012. Ecología del crecimiento de una lagartija del género Xenosaurus Peters 1861 (Squamata: Xenosauridae) en la Reserva de la Biósfera, Sierra Gorda, Querétaro, México. Rev Chil Hist Nat 85: 321-333. doi: 10.4067/S0716-078X2012000300006 